\title{
Tumor marker a-fetoprotein receptor does not discriminate between benign prostatic disease and prostate cancer
}

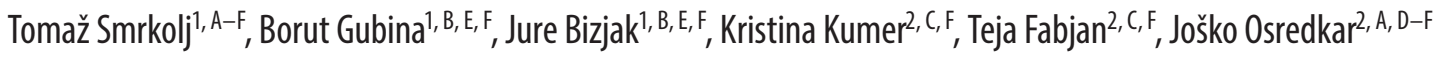 \\ 1 Department of Urology, Ljubljana University Medical Centre, Slovenia \\ ${ }^{2}$ Institute of Clinical Chemistry and Biochemistry, Ljubljana University Medical Centre, Slovenia \\ A - research concept and design; $B$ - collection and/or assembly of data; $C$ - data analysis and interpretation; \\ $D$ - writing the article; $E$ - critical revision of the article; $F$ - final approval of article
}

\section{Address for correspondence \\ Tomaž Smrkolj.}

E-mail: tomaz.smrkolj@kclj.si

\section{Funding sources}

None declared

\section{Conflict of interest}

RECAF concentration in patients' blood samples was measured free of charge by BioCurex, Inc., Suite 215, 7080 River Road, V6X 1X5 Richmond, BC, Canada. Biocurex Inc. had no role in the design of the study, the collection and analysis of the data or the decision to publish. No other conflicts of interest are declared.

\section{Acknowledgements}

The authors wish to thank BioCurex, Inc. (Canada) for supporting this study by measuring the RECAF concentrations in the patients' blood samples.

Received on April 11, 2015

Revise on August 23, 2016

Accepted on September 29, 2016

\begin{abstract}
Background. The a-fetoprotein receptor (RECAF) is a proposed novel tumor marker for detecting several different types of tumors, including prostate cancer (PCa).

Objectives. The aim of the study was to evaluate RECAF in discriminating benign prostatic conditions from PCa and to compare it with prostate-specific antigen (PSA).

Material and methods. A total of 64 patients with elevated serum PSA levels and/or abnormal digital rectal examination of the prostate referred to a tertiary center for transrectal ultrasound (TRUS) biopsy of the prostate were prospectively enrolled in the study from January 2009 to April 2010. Serum RECAF, total PSA (PPSA) and free PSA (PPSA) concentrations were measured. The results were correlated with histopathologic findings using the Mann-Whitney $U$ test and Kruskal-Wallis $x^{2}$ test.

Results. The median RECAF concentration was $5.34 \mathrm{U} / \mathrm{L}$ in the benign pathology group of patients and $4.72 \mathrm{U} / \mathrm{L}$ in the malignant pathology group. The difference was not statistically significant. RECAF density, TPSA and FPSA concentrations and IPSA density were significantly different between the benign and malignant pathology groups ( $p=0.033, p=0.000, p=0.002$ and $p=0.000$, respectively). RECAF concentration and RECAF density did not differ significantly in the subgroups of PCa patients stratified according to Gleason score, predominant primary Gleason grade or maximum primary Gleason grade, but in predominant secondary Gleason grade and maximum secondary Gleason grade, significant differences were found $(p=0.007$ and $p=0.004$, respectively).
\end{abstract}

Conclusions. The results of the study did not confirm the RECAF tumor marker as an alternative way to discriminate between groups of patients with benign prostatic conditions and $\mathrm{PC}$, and its concentration and density do not differ among PCa histopathologic groups.

Key words: prostate cancer, histopathology, prostate-specific antigen, tumor marker, a-fetoprotein receptor

DOI

$10.17219 /$ acem $/ 65432$

Copyright

Copyright by Author(s)

This is an article distributed under the terms of the

Creative Commons Attribution Non-Commercial License

(http://creativecommons.org/licenses/by-nc-nd/4.0/) 
In the last decade, prostate cancer ( $\mathrm{PCa}$ ) has become the most frequently diagnosed cancer among all solid tumors in men in developed countries. ${ }^{1}$ Its increased incidence is the result of widespread screening programs and improved awareness of the disease among the general population. The discovery of the serum tumor marker prostate-specific antigen (PSA) and its adoption in clinical practice has lowered the stage of newly discovered PCa. ${ }^{2}$ PSA is organ specific but not disease specific; it can be elevated in benign prostatic hyperplasia $(\mathrm{BPH})$, prostatitis, as well as in $\mathrm{PCa} .{ }^{3}$ PSA values in $\mathrm{BPH}$ and prostatitis often resemble PSA levels in the early stages of PCa, which is a curable disease.

A diagnosis of $\mathrm{PCa}$ is confirmed by a transrectal ultrasound (TRUS)-guided biopsy of the prostate indicated by increased PSA levels, a suspect digital rectal examination (DRE), or suspicious areas detected on a TRUS; however, up to $60 \%$ of prostate biopsy procedures still prove to be negative using these criteria. ${ }^{4-6}$ Several modifications of serum PSA value have been proposed to improve its specificity in the early detection of $\mathrm{PCa}$, for example the ratio of free PSA (fPSA) to total PSA (\%fPSA), PSA density, PSA velocity and doubling time and detection of PSA isoforms. However, their use in clinical practice is limited. ${ }^{3}$ Other molecules that may be useful as PCa markers are under investigation. Of these, prostate cancer antigen 3 (PCA3) is the most extensively studied and has already been proposed for clinical use due to its slightly better performance compared with tPSA. ${ }^{7,8}$

The $\alpha$-fetoprotein receptor (RECAF) is an oncofetal antigen present in high concentrations during fetal growth and development; however, its concentration drops to very low levels after birth and normally remains low even in adult life. ${ }^{9,10}$ It has been suggested that RECAF concentrations increase in some malignant diseases (e.g., teratocarcinoma, hepatocellular, breast, lung, prostate, ovary and gastric carcinoma), whereas in benign tumors, RECAF levels do not appear elevated. ${ }^{11-13}$

Moro et al. studied the clinical usefulness of RECAF in the diagnostics of $\mathrm{BPH}$ and $\mathrm{PCa}$, and they reported a sensitivity of $99 \%$ and a specificity of $95 \% .{ }^{11}$ However, no correlation to PSA concentration, histopathologic grading (Gleason grade), or the $\mathrm{T}$ stage of PCa was made.

The aim of the present study was to evaluate the RECAF tumor marker in the diagnostics of benign prostatic diseases and PCa and to compare it with PSA, an established clinical marker. To the authors' knowledge, this is the first study to report on correlations between RECAF and PSA and the histopathologic grading of $\mathrm{PCa}$.

\section{Material and methods}

The study was approved by the National Medical Ethics Committee of the Republic of Slovenia, and informed consent was obtained from each participant. This was a single-center study. The patients included in the study had been routinely scheduled for a TRUS-guided biopsy of the prostate by the referring urologist due to an elevated PSA concentration, suspect DRE or both. The exclusion criteria for the study were: a history of previous malignant disease, an indwelling urinary catheter or a previous positive biopsy. Any previous medical therapy for the treatment of lower urinary tract symptoms was recorded. The inclusion of the patients in the study and the TRUS-guided biopsies were performed by two certified urologists. Consecutive patients scheduled on a date on which 1 of the 2 participating urologists was performing biopsies were considered and included, if the inclusion criteria were met. While patients were scheduled for TRUS-guided biopsies up to 3 months in advance, the urologist performing the TRUS-guided biopsies was scheduled on day-to-day basis, so the patients included were a random sample. A total of 64 patients were prospectively enrolled in the study from January 2009 to April 2010. Other certified urologists and residents were performing TRUS-guided biopsies at the time of the study, but their patients were not included in the study.

\section{Detection methods}

Blood samples for analysis were obtained from each patient just before the TRUS-guided biopsies. Full blood count, serum electrolytes, alkaline phosphatase, tPSA, fPSA and RECAF markers were determined from the samples. If a patient had been taking a $5 \alpha$-reductase inhibitor for over 6 months, the tPSA and fPSA concentrations measured were doubled for the statistical analysis.

The concentration of serum RECAF was measured with a competitive chemiluminescence immunoassay (CLIA) by BioCurex Inc. (Richmond, BC, Canada). The test was a solid-phase competitive immunoassay using microwell plates in which a constant amount of RECAF-acridinium competed with the RECAF in the serum sample to bind to the monoclonal antihuman RECAF antibody immobilized in the solid phase. The amount of labeled RECAF bound to the solid phase was inversely proportional to the amount of RECAF in the sample.

PSA measurements were performed on a LIAISON CLIA analyzer (DiaSorin, Saluggia, Italy) with the LIAISON tPSA and LIAISON fPSA assays. The method for quantitative determination of tPSA and fPSA is a sandwich CLIA. The light signal, and hence the amount of isoluminol-antibody conjugate, is measured by a photomultiplier as relative light units and indicates the concentration of tPSA or fPSA present in the samples.

Before each biopsy, the clinical stage according to the DRE was noted, and the volume of the prostate was measured by TRUS examination. The TRUS-guided prostate biopsies were performed using the proto- 
Table 1. Patient characteristics stratified according to histopathologic results

\begin{tabular}{|l|c|c|}
\multicolumn{1}{|c|}{ Patient characteristics } & $\begin{array}{c}\text { Benign group median } \\
\text { (interquartile range) }\end{array}$ & $\begin{array}{c}\text { Malignant group median } \\
\text { (interquartile range) }\end{array}$ \\
\hline Number of patients & 35 & 29 \\
\hline Age (years) & $63.0(59.0-69.0)$ & $71.0(61.5-76.5)$ \\
\hline Prostate volume $(\mathrm{mL})$ & $46.0(38.0-56.8)$ & $30.0(23.0-40.1)$ \\
\hline Blood leukocytes $\left(10^{9} / \mathrm{L}\right)$ & $7.2(5.9-8.2)$ & $6.9(6.2-7.6)$ \\
\hline Hemoglobin level $(\mathrm{g} / \mathrm{L})$ & $150.0(142.0-155.0)$ & $152.0(140.5-158.0)$ \\
\hline Blood thrombocytes $\left(10^{9} / \mathrm{L}\right)$ & $210.0(176.0-262.0)$ & $230.0(207.0-288.5)$ \\
\hline Serum alkaline phosphatase $(\mu \mathrm{kat} / \mathrm{L})$ & $1.100(0.940-1.300)$ & $1.80(0.995-1.260)$ \\
\hline
\end{tabular}

Table 2. A comparison of median RECAF concentration, median PSA concentration and derived parameters between benign and malignant groups of patients

\begin{tabular}{|l|c|c|c|}
\multicolumn{1}{|c|}{ Tumor marker } & $\begin{array}{c}\text { Benign group median } \\
\text { (interquartile range) }\end{array}$ & $\begin{array}{c}\text { Malignant group median } \\
\text { (interquartile range) }\end{array}$ & Mann-Whitney U (sig.) \\
\hline Number of patients & 35 & $29^{1}$ & $472.5(p=0.637)$ \\
\hline RECAF concentration $(\mathrm{U} / \mathrm{l})$ & $5.34(3.87-9.70)$ & $4.72(3.86-9.41)$ & $349.0(p=0.033)$ \\
\hline RECAF density $\left(\mathrm{U} / \mathrm{L} / \mathrm{cm}^{3}\right)$ & $0.1187(0.0734-0.2167)$ & $0.1995(0.1344-0.3451)$ & $197.0(p=0.000)$ \\
\hline tPSA concentration $(\mu \mathrm{g} / \mathrm{L})$ & $3.57(2.40-5.10)$ & $7.71(4.58-13.50)$ & $270.0(p=0.002)$ \\
\hline fPSA concentration $(\mu \mathrm{g} / \mathrm{L})^{1}$ & $0.540(0.380-0.960)$ & $0.895(0.605-1.390)$ & $137.0(p=0.000)$ \\
\hline tPSA density $\left(\mu \mathrm{g} / \mathrm{L} / \mathrm{cm}^{3}\right)$ & $0.0795(0.0529-0.1020)$ & $0.2658(0.1199-0.5195)$ & $363.0(p=0.079)$ \\
\hline Free to total PSA ratio $(\%)^{1}$ & $18.5(10.2-24.2)$ & $13.0(8.0-18.9)$ & \\
\hline
\end{tabular}

1 Due to missing data, the number of patients in this group in rows fPSA and free to total PSA ratio is 28 ; RECAF - a-fetoprotein receptor; tPSA - total PSA: fpsa - free PSA.

col established in the authors' institution, targeting the lateral parts of the peripheral zone of both prostatic lobes.

The biopsy cores were analyzed in the histopathology laboratory of the Institute for Pathology at the Medical Faculty of Ljubljana University (Slovenia). The results were divided into benign and malignant categories and further subclassified as BPH, inflammation (prostatitis), high-grade prostatic intraepithelial neoplasia (HGPIN), suspected PCa and confirmed PCa. In the PCa reports, the Gleason grades were noted.

\section{Statistical analysis}

The data were analyzed using SPSS software (v. 21.0, IBM Corp., Armonk, USA). The measured concentrations of RECAF and PSA and their derived values were compared using the Mann-Whitney $U$ test for the 2 groups of patients. The Kruskal-Wallis $\chi^{2}$ test was used to compare the values of more than 2 groups of patients. The Kendall $\mathrm{\tau}$-b test was used to calculate the statistical correlation between PSA and RECAF concentrations and RECAF density.

\section{Results}

Complete data was obtained from 63 out of the 64 patients; in 1 patient, the fPSA concentration was not measured. Table 1 shows a comparison of general patient data stratified into benign and malignant pathology groups, in which the benign group consisted of the patients whose pathology report diagnosed $\mathrm{BPH}$, prostatitis or HGPIN, and the malignant group consisted of patients with either a suspicious report for PCa or confirmed PCa.

The median RECAF concentration was 5.34 U/L (3.879.70) in the benign pathology group and 4.72 U/L (3.869.41) in the malignant pathology group. The difference was not statistically significant (Mann-Whitney U test $=472.5 ; \mathrm{p}=0.637)$. However, when RECAF concentration was normalized to prostate volume (RECAF density), a significant difference was found between the 2 pathology groups $(\mathrm{p}=0.033)$. The tPSA and fPSA concentrations and tPSA density were also significantly different between the benign and malignant pathology groups, whereas \%fPSA was not (Table 2).

The group of patients diagnosed with $\mathrm{PCa}$ was further stratified according to the following histologic pa- 
rameters: Gleason score (Gsum), predominant primary Gleason grade (Gprim), predominant secondary Gleason grade $(\mathrm{Gsec})$, maximum primary Gleason grade (maxGprim), and maximum secondary Gleason grade (maxGsec), where predominant Gleason grade refers to the predominant Gleason grade reported by a pathologist after considering all the biopsy cores, and maximum grade refers to the highest Gleason grade found in any of the biopsy cores. The median RECAF concentrations, RECAF densities, tPSA concentrations, fPSA concentrations, PSA densities and \%fPSA were statistically com- pared among these subgroups. Out of 29 patients diagnosed with $\mathrm{PCa}, 28$ had complete histopathologic data reported; in 1 patient the exact pathologic grading could not be determined due to the small amount of $\mathrm{PCa}$ in his biopsy cores (Table 3).

No statistically significant correlations were found between tPSA and fPSA concentrations on one hand, and RECAF concentration and density on the other hand (Table 4).

Positive statistically significant correlations were found between the age of the patient and tPSA and fPSA con-

Table 3. A comparison of median RECAF concentration, median total and free PSA concentration and derived parameters between histopathologic subgroups in the patients with prostate cancer

\begin{tabular}{|c|c|c|c|c|c|c|c|}
\hline $\begin{array}{l}\text { Gleason } \\
\text { category }\end{array}$ & Gleason value & $\begin{array}{c}\text { RECAF } \\
\text { concentration } \\
(\mathrm{U} / \mathrm{L})\end{array}$ & $\begin{array}{l}\text { RECAF density } \\
\left(\mathrm{U} / \mathrm{L} / \mathrm{cm}^{3}\right)\end{array}$ & $\begin{array}{c}\text { tPSA } \\
\text { concentration } \\
(\mu \mathrm{g} / \mathrm{L})\end{array}$ & $\begin{array}{c}\text { fPSA } \\
\text { concentration } \\
(\mu \mathrm{g} / \mathrm{L})\end{array}$ & $\begin{array}{l}\text { tPSA density } \\
\left(\mu \mathrm{g} / \mathrm{L} / \mathrm{cm}^{3}\right)\end{array}$ & $\begin{array}{l}\text { Free to total } \\
\text { PSA ratio (\%) }\end{array}$ \\
\hline no. of patients & & 28 & 28 & 28 & 271 & 28 & 271 \\
\hline \multirow{5}{*}{ Gsum } & 6 & 9.42 & 0.28 & 4.42 & 0.62 & 0.152 & 18.6 \\
\hline & 7 & 5.105 & 0.215 & 8.005 & 0.895 & 0.414 & 11.4 \\
\hline & 8 & 8.025 & 0.324 & 10.94 & 0.90 & 0.361 & 9.0 \\
\hline & 9 & 4.42 & 0.123 & 15.45 & 1.78 & 0.558 & 13.4 \\
\hline & $\begin{array}{c}\text { Kruskal Wallis X² } \\
\text { (sig.) }\end{array}$ & $\begin{array}{c}6.539 \\
(p=0.088)\end{array}$ & $\begin{array}{c}6.850 \\
(p=0.077)\end{array}$ & $\begin{array}{c}12.057 \\
(p=0.007)\end{array}$ & $\begin{array}{c}9.859 \\
(p=0.020)\end{array}$ & $\begin{array}{c}9.389 \\
(p=0.025)\end{array}$ & $\begin{array}{c}3.496 \\
(p=0.321)\end{array}$ \\
\hline \multirow{3}{*}{ Gprim } & 3 & 6.245 & 0.206 & 5.395 & 0.765 & 0.199 & 14.7 \\
\hline & 4 & 4.42 & 0.168 & 13.50 & 1.60 & 0.558 & 11.0 \\
\hline & $\begin{array}{c}\text { Mann Whitney U } \\
\text { (sig.) }\end{array}$ & $\begin{array}{c}68.000 \\
(p=0.194)\end{array}$ & $\begin{array}{c}83.000 \\
(p=0.546)\end{array}$ & $\begin{array}{c}34.000 \\
(p=0.004)\end{array}$ & $\begin{array}{c}35.000 \\
(p=0.009)\end{array}$ & $\begin{array}{c}29.000 \\
(p=0.002)\end{array}$ & $\begin{array}{c}69.000 \\
(p=0.348)\end{array}$ \\
\hline \multirow{4}{*}{ Gsec } & 3 & 9.29 & 0.336 & 5.57 & 0.68 & 0.214 & 18.0 \\
\hline & 4 & 6.245 & 0.176 & 6.445 & 0.815 & 0.255 & 11.5 \\
\hline & 5 & 4.32 & 0.103 & 15.40 & 1.57 & 0.454 & 11.9 \\
\hline & $\begin{array}{c}\text { Kruskal Wallis } X^{2} \\
\text { (sig.) }\end{array}$ & $\begin{array}{c}4.555 \\
(p=0.103)\end{array}$ & $\begin{array}{c}10.063 \\
(p=0.007)\end{array}$ & $\begin{array}{c}9.199 \\
(p=0.01)\end{array}$ & $\begin{array}{c}8.057 \\
(p=0.018)\end{array}$ & $\begin{array}{c}4.356 \\
(p=0.113)\end{array}$ & $\begin{array}{c}2.216 \\
(p=0.330)\end{array}$ \\
\hline \multirow{3}{*}{ maxGprim } & 3 & 0.6245 & 0.206 & 5.395 & 0.765 & 0.199 & 14.7 \\
\hline & 5 & 4.52 & 0.143 & 10.81 & 1.40 & 0.415 & 23.4 \\
\hline & $\begin{array}{c}\text { Kruskal Wallis } x^{2} \\
\text { (sig.) }\end{array}$ & $\begin{array}{c}1.694 \\
(p=0.429)\end{array}$ & $\begin{array}{c}0.908 \\
(p=0.635)\end{array}$ & $\begin{array}{c}9.234 \\
(p=0.010)\end{array}$ & $\begin{array}{c}6.894 \\
(p=0.032)\end{array}$ & $\begin{array}{c}10.990 \\
(p=0.004)\end{array}$ & $\begin{array}{c}0.995 \\
(p=0.608)\end{array}$ \\
\hline \multirow{4}{*}{ maxGsec } & 3 & 0.929 & 0.336 & 5.57 & 0.68 & 0.214 & 18.0 \\
\hline & 4 & 0.595 & 0.205 & 6.47 & 0.87 & 0.281 & 11.0 \\
\hline & 5 & 4.145 & 0.099 & 14.55 & 1.78 & 0.447 & 13.4 \\
\hline & $\begin{array}{c}\text { Kruskal Wallis } x^{2} \\
\text { (sig.) }\end{array}$ & $\begin{array}{c}4.802 \\
(p=0.091)\end{array}$ & $\begin{array}{c}11.102 \\
(p=0.004)\end{array}$ & $\begin{array}{c}7.784 \\
(p=0.020)\end{array}$ & $\begin{array}{c}8.219 \\
(p=0.016)\end{array}$ & $\begin{array}{c}3.136 \\
(p=0.208)\end{array}$ & $\begin{array}{c}2.136 \\
(p=0.344)\end{array}$ \\
\hline
\end{tabular}

${ }^{1}$ Due to missing data, the number of patients in this group in columns fPSA and free to total PSA ratio is 27; Gsum - Gleason score;

Gprim - predominant primary Gleason grade; Gsec - predominant secondary Gleason grade; maxGprim - maximum primary Gleason grade; maxGsec - maximal secondary Gleason grade; RECAF - a-fetoprotein receptor; tPSA - total PSA; fPSA - free PSA. 
Table 4. Statistical correlation between PSA and RECAF

\begin{tabular}{|c|c|c|}
\hline Kendall $\mathrm{T}-\mathrm{b}$ (sig.) & RECAF concentration & RECAF density \\
\hline Total PSA & $-0.049(0.570)$ & $0.074(0.388)$ \\
\hline Free PSA & $0.017(0.840)$ & $-0.034(0.691)$ \\
\hline
\end{tabular}

RECAF - a-fetoprotein receptor; tPSA - total PSA; fPSA - free PSA.

centrations and PSA density, but no statistically significant correlations were found between the age of the patient and RECAF concentration and density (Table 5).

\section{Discussion}

This study focused on a comparison of novel tumor marker RECAF vs PSA, a tumor marker that has been established for PCa for 2 decades. ${ }^{2}$ A previous study reported a sensitivity of $99 \%$ and specificity of $95 \%$ for RECAF. ${ }^{11}$ However, the present study found no statistically significant difference in RECAF concentration between the benign and malignant pathology groups. Consequently, the sensitivity and specificity of the RECAF tumor marker were not determined. The median RECAF concentration was lower in the patients with PCa than in the benign pathology group. Since it has been suggested that RECAF, as an oncofetal protein, is expressed in large quantities in physiologic and pathologic states of high cell division and turnover rates, such as fetal development and some cancers, higher concentrations of RECAF in the PCa patients than in the benign pathology group were expected. ${ }^{10-13}$ On the other hand, the median RECAF density was almost 1.7 times higher in the patients with PCa than in the group with benign conditions, which was a statistically significant difference. It should be noted that the median prostatic volume was almost 1.6 times lower in the malignancy group than in the benign group, possibly introducing a bias. A much larger difference in RECAF density was expected, because RECAF concentrations in benign conditions have been reported to be nearly the same as in healthy men, suggesting a very low concentration in benign prostatic conditions. ${ }^{11}$ In contrast, the PSA measurements in the present study showed significantly higher tPSA and fPSA concentrations and tPSA density in the group of patients with $\mathrm{PCa}$ when compared with the benign group.

The age of the patient is a proven risk factor for PCa. The present study has shown a positive statistical correlation between age and tPSA, fPSA and PSA density, which were higher in the group of $\mathrm{PCa}$ patients, who were significantly older than those in the benign group.
No such correlation was found for RECAF concentration or RECAF density. The positive correlation between PSA density and age underscores the fact that in older men, PSA produced per volume of prostatic tissue increases due to $\mathrm{PCa}$ and not due to benign prostatic enlargement.

Generally, tPSA concentration is higher in PCa patients with a higher Gsum. ${ }^{14,15}$ The results of the present study show significantly higher median tPSA and fPSA concentrations in the subgroups of patients with increasing Gsum, Gprim, Gsec and maxGsec. Similarly, tPSA density also significantly increases in patients with increasing Gprim, implying higher prostatic cell division and turnover rates in patients with higher grade cancer. In contrast, RECAF concentration and RECAF density do not differ significantly in the subgroups of patients stratified according to Gsum, Gprim, and maxGprim; it was only in the subgroups with increasing Gsec and maxGsec that significant differences in RECAF density were found. Median RECAF concentrations decrease with increasing Gprim, Gsec, and maxGsec; median RECAF density decreases with increasing Gprim, Gsec, maxGprim, and maxGsec, even though one would expect that a higher rate of cell division and turnover in higher grade cancers would be reflected in higher RECAF concentration and density.

The results of this study did not confirm that higher tPSA and fPSA concentrations imply higher RECAF concentration and RECAF density, because no statistically significant correlations were found in the entire study group. As this study is the first to report on the RECAF marker in relation to the histopathologic grading of PCa and correlations to PSA, there are no other studies to compare the results with.

In conclusion, the results of the present study did not show RECAF to be an alternative tumor marker for discrimination between groups of patients with benign prostatic conditions and $\mathrm{PCa}$. Its concentration and density do not correlate with either tPSA or fPSA, which are established markers for PCa and which differ significantly between the benign and malignant pathology groups of patients in the study. In contrast to tPSA and fPSA, RECAF concentration does not significantly differ between histopathologic subgroups according to Gsum, Gprim, and Gsec grades or maxGprim and maxGsec grades. RECAF density is, paradoxically, lower in higher-end Gleason grade and maxGsec grade subgroups. To elucidate the issue of the RECAF marker in different histopathologic groups, it is necessary to conduct further studies including histopathologic staining of prostate biopsy cores using a tissue-section staining kit approved by the US Food and Drug Administration. ${ }^{11}$

Table 5. Statistical correlation between PSA and RECAF with the age of the patient

\begin{tabular}{|c|c|c|c|c|c|}
\hline Kendall T-b (sig.) & tPSA & fPSA & PSA density & RECAF concentration & RECAF density \\
\hline Age & $0.245(0.005)$ & $0.306(0.001)$ & $0.213(0.015)$ & $-0.149(0.088)$ & $-0.015(0.866)$ \\
\hline
\end{tabular}

RECAF - a-fetoprotein receptor; tPSA - total PSA; fPSA - free PSA. 


\section{References}

1. Boyle P, Ferlay J. Cancer incidence and mortality in Europe, 2004. Ann Oncol. 2005;16:481-488.

2. Stamey TA, Yang N, Hay AR, McNeal JE, Freiha FS, Redwine E. Prostate-specific antigen as a serum marker for adenocarcinoma of the prostate. N Engl J Med. 1987;317:909-916.

3. Heidenreich A, Bellmunt J, Bolla M, et al. EAU guidelines on prostate cancer. Part 1: Screening, diagnosis, and treatment of clinically localised disease. Eur Urol. 2011;59:61-71.

4. Hara R, Jo Y, Fujii T, et al. Optimal approach for prostate cancer detection as initial biopsy: Prospective randomized study comparing transperineal versus transrectal systematic 12-core biopsy. Urology. 2008;71:191-195.

5. Okotie OT, Roehl KA, Han M, Loeb S, Gashti SN, Catalona WJ. Characteristics of prostate cancer detected by digital rectal examination only. Urology. 2007;70:1117-1120.

6. Catalona WJ, Richie JP, Ahmann FR, et al. Comparison of digital rectal examination and serum prostate specific antigen in the early detection of prostate cancer: Results of a multicenter clinical trial of 6,630 men. J Urol. 1994;151:1283-1290.

7. Deras IL, Aubin SM, Blase A, et al. PCA3: A molecular urine assay for predicting prostate biopsy outcome. J Urol. 2008;179:1587-1592.

8. Hessels D, Klein Gunnewiek JM, van Oort I, et al. DD3(PCA3)-based molecular urine analysis for the diagnosis of prostate cancer. Eur Urol. 2003;44:8-15.

9. Moro R, Tamaoki T, Wegmann TG, Longenecker BM, Laderoute MP. Monoclonal antibodies directed against a widespread oncofetal antigen: The alpha-fetoprotein receptor. Tumour Biol. 1993;14:116-130.

10. Newby D, Dalgliesh G, Lyall F, Aitken DA. Alphafetoprotein and alphafetoprotein receptor expression in the normal human placenta at term. Placenta. 2005;26:190-200.

11. Moro R, Tcherkassova J, Song E, et al. A new broad-spectrum cancer marker. IVD Technology Magazine 2005;59.

12. Tsuboi S, Taketa K, Nouso K, et al. High level of expression of alphafetoprotein receptor in gastric cancers. Tumour Biol. 2006;27:283-288.

13. Moro R, Gulyaeva-Tcherkassova J, Stieber P. Increased alpha-fetoprotein receptor in the serum of patients with early-stage breast cancer. Curr Oncol. 2012;19:e1-8.

14. Lekili M, Zengin M, Postaci H, Ayder AR. Relationship between histologic grading and serum prostate specific antigen in prostatic carcinoma. Int Urol Nephrol. 1994;26:665-668.

15. Douglas TH, Morgan TO, McLeod DG, et al. Comparison of serum prostate specific membrane antigen, prostate specific antigen, and free prostate specific antigen levels in radical prostatectomy patients. Cancer. 1997;80:107-114. 